\title{
Uma chama que se apaga: residência de Medicina Preventiva e Social
}

\section{A failing ligth: preventive and social medical residence}

\author{
Adriano Massuda ${ }^{\mathrm{I}}$ \\ Nelson Filice de Barros ${ }^{\text {II }}$ \\ Roberto Jorge Freire Esteves ${ }^{\mathrm{I}}$ \\ Viviane Ribeiro Guimarães ${ }^{\mathrm{I}}$
}

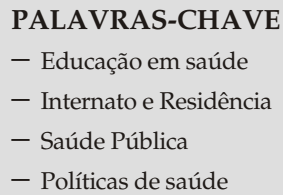

\section{KEY WORDS}

- Health Education

- Internship and Residency

- Public Health

- Health politics
Recebido em: 15/12/2008

Reencaminhado em: 03/03/2009

Aprovado em: 24/03/2009

\section{R E S U M O}

O processo de extinção da residência de Medicina Preventiva e Social (RMPS) constitui um paradoxo diante da necessidade de médicos com especialização em Saúde Coletiva para atuar no Sistema Único de Saúde. O presente artigo realizou uma revisão do processo histórico de construção das RMPS e investigou a estrutura atual dos programas remanescentes a partir da análise dos cenários de prática e das atividades práticas e teóricas desenvolvidas. Ao final foi possível construir uma tipologia em que se identifica o tipo Generalista em Saúde Coletiva, que aborda as três áreas nucleares da Saúde Coletiva; o tipo da Epidemiologia, no qual o foco é exclusivamente essa área; e o tipo da Saúde Comunitária, que se centra na Atenção Primária em Saúde. Do cruzamento do processo histórico de construção com a realidade atual, pode-se inferir que os fatores que têm contribuído para a extinção da RMPS são principalmente a ausência de políticas de Estado para formação na área, bem como o não comprometimento de sujeitos sociais que se relacionam com essa residência, levando à existência de anacrônicas diretrizes orientadoras para formação profissional.

\section{A B S T R A C T}

The extinction process of Preventive and Social Medicine Residency Programs (PSMR) have experienced constitutes a paradox in the face of the need for physicists with Collective Health Specialization in the Unified Health System. The present paper investigated which are the practice sceneries and how the practical and theoretical activities are developed into PSMR programs. It was observed that most programs include actions in health assistance, as well as activities in the three main fields of Collective Health. However, significant differences were evidenced among the programs. It was possible construct a typology, identifying the type 'Generalist in Collective Health', who approaches the three main fields of Collective Health; the type 'Epidemiology', which focuses exclusively on this field; and the type 'Community Health', which is centered in Health Basic Care. From the crossing among historical construction process and present reality, it was possibly infer that the inexistent of State Politics to graduate health professionals in this area, and not compromise of involved social subjects with this residence are the most important factors, resulting in the existence of an anachronism in the guidelines. 


\section{INTRODUÇÃO}

A residência de Medicina Preventiva e Social (RMPS) constitui uma área de especialidade, reconhecida pela Comissão Nacional de Residência Médica (CNRM), que se destina à formação de médicos na área da Saúde Pública/Saúde Coletiva. Até o início de 2007, quando foi finalizada a pesquisa que serviu de base para a preparação deste artigo, existiam apenas 11 programas com residentes inscritos e não mais que 20 residentes matriculados por ano. Número bastante aquém da dimensão que a RMPS apresentou em seu período áureo, no início da década de 1980, quando chegou a ter mais de 180 residentes inscritos por ano ${ }^{1}$.

Pode-se se inferir que a progressiva diminuição do número de residentes de RMPS verificada nos últimos anos esteja correlacionada com a redução da importância dessa residência na formação de quadros técnicos para o Sistema Único de Saúde (SUS), bem como na formação profissional no campo da Saúde Coletiva. Contexto bastante distinto dos anos que marcaram a conformação do campo da Saúde Coletiva e que antecederam a construção e implantação do SUS, quando os egressos dos programas de RMPS constituíam os quadros técnicos que compunham a base do movimento sanitário brasileiro ${ }^{2,3,4}$.

Paradoxalmente, é após a implantação do SUS e a afirmação da Saúde Coletiva como campo especializado de conhecimento que se observa o início da trajetória que tem apontado a extinção da RMPS num futuro próximo. Momento em que seria esperada a ampliação da necessidade de médicos com esse tipo de formação, seja para renovar os quadros do movimento sanitário como um ator político participante da construção das políticas de saúde, seja para a inserção profissional em diferentes funções nos serviços de saúde e na organização do Sistema de Saúde, em que deveria se exigir formação técnica no campo da Saúde Coletiva.

Com o intuito de incitar uma reflexão a respeito de outros caminhos para as RMPS que não a extinção, apresenta-se o resultado de uma pesquisa que buscou compreender o atual estado dos programas de RMPS remanescentes no Brasil, correlacionando-o com os fatores determinantes do processo histórico de construção dessas residências.

\section{METODOLOGIA}

Este estudo é um dos produtos finais do projeto de Caracterização dos Programas de Residência em Medicina Preventiva e Social no Brasil, desenvolvido por membros do Laboratório de Pesquisa Qualitativa em Saúde, do Departamento de Medicina Preventiva e Social, da Universidade Estadual de Campinas, em colaboração com o Observatório de Recursos Humanos em Saúde de São Paulo (ObservaRHSP). Outras informações podem ser obtidas no sítio http://www.observarhsp.org.br/projetos/formacao.html.

A pesquisa foi desenvolvida em duas etapas: na primeira, foi realizada uma revisão da literatura sobre o tema e pesquisa com dados secundários a fim de identificar os programas de RMPS existentes e com residentes matriculados; na segunda, realizou-se um levantamento de dados primários, com aplicação de questionário semiestruturado, por telefone, e entrevista em profundidade in loco.

Os questionários foram enviados por correio eletrônico às instituições e em seguida foram realizadas entrevistas telefônicas para conferir as informações registradas nos questionários. As entrevistas, por sua vez, foram realizadas com vistas a aprofundar as questões levantadas nos questionários. Ao todo, trabalhou-se com 11 programas de residência, o que corresponde ao total de programas de RMPS com residentes inscritos no ano de 2006, e, desses, dez participaram de todas as etapas da investigação.

Para descrição da estrutura organizacional dos programas, trabalhou-se com o reconhecimento de três categorias mediante a aplicação dos questionários: cenários de práticas onde são realizadas as atividades, atividades práticas desenvolvidas pelos residentes durante o programa de residência e conteúdos teóricos oferecidos no programa.

Para estabelecer uma matriz comparativa, utilizaram-se os requisitos mínimos para o funcionamento dos programas da RMPS definidos pela CNRM, segundo a sua Resolução 02/2006. Os Quadros 1,2 e 3 apresentam, respectivamente, o que estabelece a Resolução CNRM 02/2006 quanto aos cenários de práticas, atividades práticas e abordagem teórica. Entretanto, em virtude da desatualização dessa Resolução, foram necessários ajustes para contemplar itens que ela não previa.

Quadro 1

Cenários de práticas em programas de RMPS - CNRM

- Técnico-operacional: unidades de prestação de cuidados de saúde - posto de saúde, centro de saúde, unidade mista e hospital

- Técnico-administrativo: Secretaria Estadual de Saúde, Secretaria Municipal de Saúde, Instituto Nacional de Assistência Médica e Previdência Social

- Político-institucional: organismos e representações institucionais e lideranças sociais que constituem poder decisório sobre questões de saúde 
Quadro 2

Atividades práticas em programas de RMPS - CNRM

- Ações de vigilância epidemiológica e epidemiologia clínica

- Elaboração e/ou análise de diagnósticos de nível de saúde e de sistema de prestação de serviços de saúde

- Elaboração e/ou análise de planos e programas de saúde para níveis local e regional

- Análise de planos e programas de saúde para níveis estadual e nacional

- Participação em atividades de administração em nível local, regional e/ou central

- Participação em programas de prestação de recursos humanos para a saúde

- Participação em atividades de órgãos ou serviços de saúde ocupacional

- Realização de atividades em programas de cuidados básicos de saúde e/ou outros programas prioritários de assistência médica, tais como saúde materno-infantil, controle de doenças transmissíveis, saúde mental e doenças degenerativas

Quadro 3

Conteúdos teóricos em programas de RMPS - CNRM
- Epidemiologia
- Administração e planejamento
- Educação em saúde e desenvolvimento de recursos humanos
- Saúde ocupacional e ambiental
- Investigação em saúde coletiva
- Ciências sociais
- Prestação de serviços básicos de saúde

Este estudo foi elaborado de acordo com os princípios éticos enunciados na Declaração de Helsinque III. As informações colhidas foram mantidas em sigilo, e o protocolo de pesquisa aprovado pelo Comitê de Ética em Pesquisa da FCM/Unicamp.

\section{HISTÓRICO DOS PROGRAMAS DE RMPS}

O processo de construção das RMPS se dá no Brasil entre as décadas de 1960 e 1980, em meio a um período em que se destacam três importantes eventos: a conformação do campo da Saúde Coletiva, a construção dos conceitos e princípios que formam a base das políticas de saúde que dão origem ao SUS e a institucionalização da residência médica como modalidade de especialização médica.

A residência médica constitui uma modalidade de formação destinada à especialização, em que o ensino é baseado fundamentalmente na prática, sendo a oferta teórica complementar à formação. Foi desenvolvida inicialmente nos Estados Unidos por volta da metade do século 19 e implantada no Brasil entre as décadas de 1940 e 1950. Expandiu-se, a partir de então, seguindo a lógica de orientação do modelo assistencial hegemônico no
País, médico-liberal, que apresentava o hospital como principal cenário de práticas ${ }^{5}$.

A RMPS, por sua vez, fundamenta-se no mesmo modelo de ensino baseado na prática, mas se distingue fundamentalmente dos outros programas por apresentar especificidades como a necessidade de um amplo aporte teórico de diferentes campos do conhecimento, entre eles: ciências sociais, epidemiologia e estatística, além da política, planejamento e gestão. Além disso, apresenta a necessidade de inserção dos residentes em diferentes cenários de práticas que não o hospital e mantém estreita ligação com as políticas de saúde em função da necessidade de desenvolvimento de atividades em cenários técnico-administrativos e político-institucionais.

No Brasil, o primeiro programa de RMPS foi criado em 1962, ainda com o nome de Medicina Preventiva, na Faculdade de Medicina de Ribeirão Preto, seguido da Universidade de São Paulo, em 1968, e da Santa Casa de São Paulo, um ano após. A implantação desses programas se deu em decorrência da criação dos Departamentos de Medicina Preventiva nas Faculdades de Medicina, sob influência da proposta formulada pelo "movimento preventivista" ${ }^{\prime 2}$. 
A proposta "preventivista", desenvolvida nos Estados Unidos entre as décadas de 1930 e 1940, buscava a reforma da prática médica a partir de mudanças localizadas na formação médica, sendo a incorporação de disciplinas de Medicina Preventiva nos currículos de Medicina a principal estratégia para atingir esse objetivo. Essas propostas foram divulgadas para a América Latina em seminários organizados pela Organização Pan-Americana de Saúde (Opas), na metade da década de $1950^{6}$.

Entretanto, as orientações liberais e a limitação da proposta "preventivista" foram logo evidenciadas ${ }^{6}$. Da crítica ao preventivismo, fundamentada em parte no resgate das proposições da Medicina Social ${ }^{7}$, e da incorporação crítica da Saúde Pública tradicional, conformou-se o marco teórico sobre o qual veio a se constituir a Medicina Preventiva e Social ${ }^{8}$, denominação com que foi registrado o programa de residência em meio ao processo de regulamentação da residência médica, ocorrido no final da década de 1970 e início dos anos $1980^{5}$.

Até meados da década de 1970, a RMPS teve um papel irrelevante na relação com as políticas de saúde no País. Sua função estava mais direcionada à formação de docentes para atuar nos departamentos de Medicina Preventiva. Nessa época, houve um ligeiro aumento do número de programas de residência na área, sem que ocorressem modificações substanciais na configuração deste tipo de formação. Em 1977, das 76 escolas médicas existentes no País, somente 13 tinham programas de RMPS, estando a oferta de vagas limitada a não mais que $20^{9}$.

Apenas no final dos anos 1970 e início dos 1980 é que a RMPS passa a ter uma importância maior na relação com a política de saúde do País. Estudos realizados pelo Instituto Nacional de Medicina e Previdência Social (Inamps) estimaram a necessidade de 1.800 profissionais com formação em Medicina Preventiva e Social para atuar nas áreas de gestão, planejamento e epidemiologia. Os programas de RMPS recebiam recursos da Previdência Social para a ampliação da oferta de vagas.

Entretanto, apesar da avaliação dessa necessidade, o Governo Federal determinou ao Inamps que cortasse todas as bolsas das RMPS, mantendo apenas aquelas cujos programas se transformassem em Medicina Comunitária. Diante disso, ocorreu a primeira grande mobilização da Associação Brasileira de Pós-Graduação em Saúde Coletiva (Abrasco) em defesa das RMPS, promovendo uma mudança no quadro. Como resultado, foi criada uma política pública de incentivo às RMPS, chamada de PAR - Programa de Apoio às Residências de Medicina Social, Medicina Preventiva e Saúde Pública -, que promoveu um considerável aumento do número de vagas para 130, em 1980, e 187, em $1981^{9}$.
Durante o período em que vigorou o PAR, intensificaram-se os debates a respeito dos objetivos e do campo de ação dos profissionais da Saúde Coletiva, chegando a ocorrer uma ruptura entre duas tendências: uma que deu origem à residência em Medicina Geral e Comunitária, com o objetivo de formar um médico generalista, cujo treinamento teria ênfase na Atenção Primária em Saúde; e outra que permaneceu como RMPS, objetivando formar profissionais com a perspectiva coletiva do cuidado, articulando práticas socialmente estruturadas e determinantes sociais do processo saúde-doença² ${ }^{2}$.

Ao longo da década de 1990, a tendência da Medicina Geral e Comunitária foi reforçada e modificada com a implantação do Programa de Saúde da Família (PSF) como estratégia estruturante da Atenção Primária no Brasil e com a incorporação de outros referenciais internacionais relativos ao papel e à formação de médicos de família, principalmente canadenses ${ }^{10}$. Com isso, essa tendência tornou-se reconhecida como um novo e distinto programa de residência pela CNRM, que passou a ser denominado Medicina de Família e Comunidade (MFC), sendo estimulada a abertura de novos programas e a ampliação de vagas como estratégia para a formação de profissionais na área.

A RMPS, por sua vez, manteve-se associada à conformação do campo da Saúde Coletiva, que Donnangelo descreveu como
uma especialidade de conhecimento, contribuindo para a ampliação da capacidade de compreensão e interven- ção sobre os determinantes do processo saúde-doença; um campo de atuação política, subsidiando a conforma- ção de modelos de atenção à saúde e a construção de po- líticas públicas de saúde capazes de apoiar mudanças no perfil de morbi-mortalidade brasileiro; e um campo am- pliado de saberes e práticas, eminentemente interdisci- plinar e multiprofissional, composto pelas áreas de Epi- demiologia, Ciências Sociais e Política, Gestão e Planeja- mento $^{11}$

O período que marca a ascensão da proposta da MFC é o mesmo da decadência da RMPS. A partir da década de 1990, e com maior ênfase a partir do ano 2000, ocorre uma diminuição progressiva na procura por parte dos egressos dos cursos de Medicina pela RMPS, com o fechamento de programas tradicionais ou a transfiguração em MFC ou Saúde Ocupacional ${ }^{1}$.

Nesse período, não se verifica a tomada de nenhuma medida efetiva por parte do Estado, das universidades ou da sociedade civil organizada no sentido de promover mudanças estruturais nos programas de RMPS ou de incentivar a formação na área da Saúde Coletiva por essa via.

Em um dos únicos eventos encontrados em que se discutiu o tema, em seminário organizado pela CNRM em 1999², bus- 
cou-se definir o perfil de saberes, de práticas e de competências a serem desenvolvidos pelas RMPS. Entretanto, não se chegou a produzir mudanças nos requisitos para o funcionamento desses programas, os quais foram reeditados pela CNRM em 2006, sem nenhuma mudança em relação à primeira edição produzida em 1981. De fato, a colagem é tão fiel que se chega a propor que o extinto Instituto Nacional de Assistência Médica e Previdência Social (Inamps) seja campo de prática para a residência.

\section{A ESTRUTURA DOS PROGRAMAS DE RMPS REMANESCENTES}

Dos mais de 20 programas de RMPS que já existiram no Bra$\mathrm{sil}^{2}$, este estudo verificou a remanescência de apenas 11 com residentes inscritos no ano de 2006. São eles: FM-ABC, HSE-RJ, IAMSPE, UFBA, UFF, UFPB, UFPE, Unicamp, UPE, FM-USP e Unesp.

Em relação à estrutura desses programas, no tocante aos cenários utilizados para os residentes realizarem suas atividades práticas, verificou-se, de maneira geral, concentração em cenários técnico-assistenciais, conforme demonstrado no Quadro 4 .
Nesses cenários ocorre concentração de atividades especialmente em serviços de atenção primária (9), dos quais em dois programas se utilizam Centros de Saúde Escola. Observou-se ainda que os hospitais constituem um importante cenário de práticas (8) e em menor número são utilizados centros de referência e/ou atenção especializada (4).

A respeito dos cenários técnico-administrativos, destaca-se a utilização de secretarias municipais (7) e, em menor número, distritos de saúde (5). Em número bastante discreto foram referidos estágios em secretaria estadual (2), Ministério da Saúde e agências reguladoras (1). E em apenas um programa foi verificada a realização de atividades em cenários político-institucionais: conselhos de saúde (1).

Destaca-se, de maneira importante, que a inserção dos residentes nos cenários de práticas se dá, essencialmente, ao longo do primeiro ano do programa. Em alguns, o estágio se repete no segundo ano em serviços de atenção primária (2), hospital (3) e distrito de saúde (1). E, em poucos cenários, são realizados estágios exclusivamente no segundo ano da residência, como secretarias de saúde (2), hospital (1) e centros de especialidade (1).

Quadro 4

Tipos de cenários de práticas por programa de RMPS

\begin{tabular}{|c|c|c|c|c|c|c|c|c|c|c|c|c|c|c|c|c|c|c|c|c|c|c|}
\hline \multirow[t]{3}{*}{ Cenários de práticas } & \multicolumn{2}{|c|}{$\begin{array}{l}\text { FM- } \\
\text { ABC }\end{array}$} & \multicolumn{2}{|c|}{$\begin{array}{l}\text { HSE- } \\
\text { RJ }\end{array}$} & \multicolumn{2}{|c|}{$\begin{array}{l}\text { IAMS } \\
\text { PE }\end{array}$} & \multicolumn{2}{|c|}{ UFBA } & \multicolumn{2}{|c|}{ UFF } & \multicolumn{2}{|c|}{ UFPB } & \multicolumn{2}{|c|}{$\begin{array}{c}\text { UFPE } \\
\text { L }\end{array}$} & \multicolumn{2}{|c|}{$\begin{array}{l}\text { UNIC } \\
\text { AMP }\end{array}$} & \multicolumn{2}{|c|}{ UPE } & \multicolumn{2}{|c|}{ USP } & \multicolumn{2}{|c|}{ Total } \\
\hline & $\mathrm{R}$ & $\mathrm{R}$ & $R$ & $\mathrm{R}$ & $\mathrm{R}$ & $\mathrm{R}$ & $\mathrm{R}$ & $\mathrm{R}$ & $\mathrm{R}$ & $\mathrm{R}$ & $\mathrm{R}$ & $\mathrm{R}$ & $\mathrm{R}$ & $\mathrm{R}$ & $\mathrm{R}$ & $\mathrm{R}$ & $\mathrm{R}$ & $\mathrm{R}$ & $\mathrm{R}$ & $\mathrm{R}$ & $\mathrm{R}$ & $\mathrm{R}$ \\
\hline & 1 & 2 & 1 & 2 & 1 & 2 & 1 & 2 & 1 & 2 & 1 & 2 & 1 & 2 & 1 & 2 & 1 & 2 & 1 & 2 & 1 & 2 \\
\hline $\begin{array}{l}\text { Serviços de atenção primária } \\
\text { Rede pública }\end{array}$ & & & & & $x$ & & $x$ & & $x$ & & $x$ & & $x$ & $x$ & $x$ & & $x$ & $x$ & & & 07 & 02 \\
\hline $\begin{array}{l}\text { Serviços de atenção primária } \\
\text { Centro escola }\end{array}$ & $x$ & & & & & & & & & & & & & & & & & & $x$ & & 02 & 00 \\
\hline $\begin{array}{l}\text { Centros de referência e/ou atenção } \\
\text { especializada }\end{array}$ & $x$ & & & & & $x$ & & & $x$ & & & & & & $X$ & & & & & & 04 & 01 \\
\hline Hospital & $x$ & & $x$ & $x$ & $x$ & & & & $x$ & & $x$ & $x$ & & & & $x$ & $x$ & $x$ & $x$ & & 06 & 04 \\
\hline Distrito de Saúde & & & & & & & & $x$ & & & $x$ & & & & & $x$ & $x$ & $x$ & $x$ & & 03 & 03 \\
\hline Secretaria Municipal de Saúde & $x$ & & & & $x$ & & $x$ & & $x$ & & & $x$ & & & & $x$ & & & $x$ & & 05 & 01 \\
\hline Secretaria Estadual de Saúde & & & & & $x$ & & & & $x$ & & & & & & & & & & & & 02 & 01 \\
\hline $\begin{array}{l}\text { Ministério da Saúde e agências } \\
\text { reguladoras }\end{array}$ & & & & & & & & & $x$ & & & & & & & & & & & & 01 & 00 \\
\hline Conselhos de Saúde & & & & & & & & & $x$ & & & & & & & & & & & & 01 & 00 \\
\hline Pesquisa na Universidade & & & & & & & & $x$ & & & & & & & & & $\mathrm{x}$ & $x$ & $x$ & & 02 & 02 \\
\hline $\begin{array}{l}\text { Área de concentração de escolha do } \\
\text { residente }\end{array}$ & & $x$ & & & & & & & & $x$ & & & & & & & & & & $x$ & 00 & 03 \\
\hline Total & 04 & 01 & 01 & 01 & 04 & 01 & 02 & 01 & 07 & 01 & 03 & 02 & 01 & 01 & 02 & 03 & 04 & 04 & 07 & 01 & & \\
\hline
\end{tabular}


Sobre as atividades práticas desenvolvidas pelos residentes (Quadro 5), ocorre maior concentração de atividades ligadas à assistência em programas de cuidados básicos de saúde e/ou outros programas prioritários de assistência médica (9). Também foi verificado importante número de programas que oferecem atividades de administração em saúde (8), vigilância em saúde e epidemiologia clínica (7), programas de prestação de recursos humanos para a saúde (7) e atividades ligadas à saúde ocupacional (6).
Em menor número, verificou-se a realização de atividades práticas ligadas à programação em saúde (5) e trabalho com informações em saúde para análise de diagnósticos de nível de saúde e de sistema de prestação de serviços de saúde (4). E observou-se ainda que em metade dos programas analisados são realizadas pesquisas em saúde coletiva (5).

Quadro 5

Tipos de atividades práticas desenvolvidas nos programas de RMPS

\begin{tabular}{|c|c|c|c|c|c|c|c|c|c|c|c|c|c|c|c|c|c|c|c|c|c|c|}
\hline \multirow{2}{*}{ Atividades práticas } & $\mathrm{R}$ & $\mathrm{R}$ & $\mathrm{R}$ & $\mathrm{R}$ & $\mathrm{R}$ & $\mathrm{R}$ & $\mathrm{R}$ & $\mathrm{R}$ & $\mathrm{R}$ & $\mathrm{R}$ & $\mathrm{R}$ & $\mathrm{R}$ & $\mathrm{R}$ & $\mathrm{R}$ & $\mathrm{R}$ & $\mathrm{R}$ & $\mathrm{R}$ & $\mathrm{R}$ & $\mathrm{R}$ & $\mathrm{R}$ & $\mathrm{R}$ & $\mathrm{R}$ \\
\hline & 1 & 2 & 1 & 2 & 1 & 2 & 1 & 2 & 1 & 2 & 1 & 2 & 1 & 2 & 1 & 2 & 1 & 2 & 1 & 2 & 1 & 2 \\
\hline $\begin{array}{l}\text { Programas de cuidados básicos de } \\
\text { saúde e/ou outros programas } \\
\text { prioritários de assistência médica }\end{array}$ & $X$ & & & & $x$ & & $x$ & & $x$ & & $x$ & $x$ & $X$ & $x$ & $x$ & & $x$ & $x$ & $x$ & & 09 & 03 \\
\hline $\begin{array}{l}\text { Administração em nível local, } \\
\text { regional e/ou central }\end{array}$ & $X$ & & & & $X$ & & $x$ & $x$ & $X$ & & $x$ & $X$ & & & $X$ & $X$ & $x$ & $x$ & $x$ & & 08 & 04 \\
\hline $\begin{array}{l}\text { Ações de vigilância epidemiológica e } \\
\text { epidemiologia clínica }\end{array}$ & & & $x$ & $X$ & $X$ & & $x$ & $x$ & & & $x$ & & & & $x$ & $x$ & $x$ & $X$ & $x$ & & 07 & 04 \\
\hline $\begin{array}{l}\text { Atividades de órgãos ou serviços de } \\
\text { saúde ocupacional }\end{array}$ & $x$ & & $X$ & $x$ & & $x$ & & & $x$ & & $x$ & & & & $x$ & & & & & & 06 & 01 \\
\hline $\begin{array}{l}\text { Elaboração e/ou análise de planos e } \\
\text { programas de saúde para níveis local } \\
\text { e regional }\end{array}$ & & & & & $X$ & & & & $x$ & & & & $X$ & $x$ & $x$ & $X$ & & & $x$ & & 05 & 02 \\
\hline $\begin{array}{l}\text { Elaboração e/ou análise de } \\
\text { diagnósticos de nível de saúde e de } \\
\text { sistema de prestação de serviços de } \\
\text { saúde }\end{array}$ & & & & & & & & $x$ & $x$ & & & & & & & $X$ & & & $X$ & & 02 & 02 \\
\hline Total & 03 & 01 & 02 & 02 & 05 & 01 & 03 & 04 & 07 & 01 & 05 & 04 & 03 & 03 & 06 & 06 & 04 & 04 & 07 & 01 & & \\
\hline
\end{tabular}


Por fim, no que se refere aos núcleos temáticos abordados nos programas de RMPS, observa-se, no Quadro 6, que a maioria dos programas oferece atividades teóricas complementares ao longo do primeiro ano da residência, sobretudo nas três áreas formadoras da Saúde Coletiva: epidemiologia (10), administração e planejamento em saúde (9) e ciências sociais (8).

Em menor número, são realizadas atividades com abordagem de Investigação em Saúde Coletiva (5), Educação em Saúde
(4) e Saúde Ocupacional (2). Destaca-se, ainda, a abordagem de análise institucional em apenas um dos programas analisados.

Por outro lado, chama muita atenção o fato de que uma das principais atividades práticas realizadas nos programas de RMPS - a participação em programas de cuidados básicos de saúde e/ou em outros programas prioritários de assistência médica-é abordada de forma teórica em apenas um programa na forma de prestação de serviços básicos em saúde.

Quadro 6

Núcleos temáticos abordados nos programas de RMPS

\begin{tabular}{|c|c|c|c|c|c|c|c|c|c|c|c|c|c|c|c|c|c|c|c|c|c|c|}
\hline \multirow{3}{*}{ Atividades práticas } & \multicolumn{2}{|c|}{$\begin{array}{l}\text { FM- } \\
\text { ABC }\end{array}$} & \multicolumn{2}{|c|}{$\begin{array}{l}\text { HSE- } \\
\text { RJ }\end{array}$} & \multicolumn{2}{|c|}{$\begin{array}{c}\text { IAMS } \\
\text { PE }\end{array}$} & \multicolumn{2}{|c|}{ UFBA } & \multicolumn{2}{|c|}{ UFF } & \multicolumn{2}{|c|}{ UFPB } & \multicolumn{2}{|c|}{$\begin{array}{l}\text { UFPE } \\
\text { L }\end{array}$} & \multicolumn{2}{|c|}{$\begin{array}{l}\text { UNIC } \\
\text { AMP }\end{array}$} & \multicolumn{2}{|c|}{ UPE } & \multicolumn{2}{|c|}{ USP } & \multicolumn{2}{|c|}{ Total } \\
\hline & $\mathrm{R}$ & $\mathrm{R}$ & $\mathrm{R}$ & $\mathrm{R}$ & $\mathrm{R}$ & $\mathrm{R}$ & $\mathrm{R}$ & $\mathrm{R}$ & $\mathrm{R}$ & $\mathrm{R}$ & $\mathrm{R}$ & $\mathrm{R}$ & $\mathrm{R}$ & $\mathrm{R}$ & $\mathrm{R}$ & $\mathrm{R}$ & $\mathrm{R}$ & $R$ & $\mathrm{R}$ & $\mathrm{R}$ & $\mathrm{R}$ & $\mathrm{R}$ \\
\hline & 1 & 2 & 1 & 2 & 1 & 2 & 1 & 2 & 1 & 2 & 1 & 2 & 1 & 2 & 1 & 2 & 1 & 2 & 1 & 2 & 1 & 2 \\
\hline Administração e planejamento & $x$ & & & & $x$ & & $x$ & $x$ & $x$ & & $x$ & & $x$ & $x$ & $x$ & $x$ & $x$ & $x$ & $x$ & & 09 & 04 \\
\hline Epidemiologia & $x$ & & $x$ & $x$ & $x$ & & $x$ & & $x$ & & $x$ & & $x$ & $x$ & $x$ & $x$ & $x$ & $x$ & $x$ & & 09 & 04 \\
\hline Ciências Sociais & $x$ & & & & $x$ & & $x$ & & $x$ & & $x$ & & & & $x$ & $x$ & $x$ & & $x$ & & 08 & 01 \\
\hline $\begin{array}{l}\text { Educação em saúde e } \\
\text { desenvolvimento de recursos } \\
\text { humanos }\end{array}$ & & & & & & & $x$ & & $x$ & & & & & & $x$ & & $x$ & $x$ & & & 04 & 01 \\
\hline Análise Institucional & & & & & & & & & & & & & & & $x$ & $x$ & & & & & 01 & 01 \\
\hline Saúde Ocupacional e Ambiental & & & & & & $x$ & $x$ & & & & & & & & & & $x$ & & & & 02 & 01 \\
\hline Investigação em Saúde Coletiva & & & $x$ & $x$ & & & $x$ & $x$ & $x$ & & & $x$ & & & & & $x$ & $x$ & $x$ & & 05 & 04 \\
\hline $\begin{array}{l}\text { Prestação de serviços básicos de } \\
\text { saúde }\end{array}$ & & & & & & & & & & & & $x$ & $x$ & & & & & & & & 01 & 00 \\
\hline Área de escolha do residente & & $x$ & & & & & & & & $x$ & & & & & & & & & & $x$ & 00 & 03 \\
\hline Total & 03 & 01 & 03 & 03 & 03 & 01 & 06 & 01 & 07 & 01 & 03 & 01 & 03 & 03 & 05 & 04 & 08 & 05 & 05 & 01 & & \\
\hline
\end{tabular}


Entretanto, poucas coisas são tão comuns à maioria dos programas de RMPS como a concentração de atividades práticas e teóricas no primeiro ano da residência, sendo, em geral, o segundo ano destinado à concentração de atividades em determinada área específica. Nesse sentido, um tipo de atividade específica do segundo ano que merece destaque por estar presente em três instituições, FM-ABC, UFF e USP, é o que se denomina "área de escolha do residente".

\section{DISCUSSÃO}

O cruzamento da história das RMPS com o que foi encontrado nos programas remanescentes nos permite fazer inferências em relação aos fatores determinantes do processo de construção e desconstrução desse programa, principalmente no que diz respeito à relação com a construção do campo da Saúde Coletiva e a definição das políticas públicas de saúde no País.

Ao analisar o processo histórico de construção das RMPS, destaca-se que seu surgimento tem origem a partir de uma proposta norte-americana de sistematização de um saber que objetiva promover mudanças na prática médica, contudo, sem promover reformas substanciais no modelo médico-hegemônico ou na configuração da política liberal para a saúde. Pelo contrário, a proposta "preventivista" buscava promover adaptações nas insuficiências e nos limites do modelo já apontadas no início das primeiras décadas do século 20.

No entanto, a proposta "preventivista" foi reconstruída no Brasil abrindo espaço para o surgimento de um novo sujeito coletivo, o movimento sanitário, e de um novo campo especializado do conhecimento, a Saúde Coletiva, baseado num conjunto de saberes e práticas específicas, que também atuava na disputa política pela reforma das práticas e da organização dos serviços de saúde. Nesse contexto, a RMPS era um dispositivo que servia à formação técnico-profissional no campo e também à formação de militantes da Reforma Sanitária.

Por outro lado, na análise do contexto histórico das RMPS é preciso considerar outra categoria atuante na definição do seu processo de auge e de extinção. O período áureo da RMPS, ocorrido no início dos anos 1980, se dá quando a formação nessa especialidade é reconhecida pelo Estado, por meio do Inamps, como necessária à construção do Sistema de Saúde brasileiro e conta com apoio e mobilização de um sujeito social, Abrasco, passando a ser estimulada pelo PAR. A política de formação, por sua vez, estava articulada com a criação do cargo e da carreira de sanitarista para incorporar esse profissional formado ao quadro técnico estatal.
O início do processo de extinção das RMPS coincide com a adoção de diretrizes neoliberais no País, a partir do início dos anos 1990, que passam a orientar as políticas públicas para a saúde e a nova conformação do Estado brasileiro ${ }^{13}$. Tais diretrizes apresentavam como objetivos a redução do tamanho do Estado e a focalização dos programas sociais, buscando reduzir o gasto público em nome do ajuste econômico ${ }^{14}$.

A adoção de diretrizes neoliberais, ainda incrustadas no ideário político-econômico do País, pode ajudar a explicar o contexto que levou à despreocupação, ou à omissão, do Estado brasileiro com a formação de uma inteligência burocrática para ocupar espaços estratégicos da gestão do sistema público de saúde. Esses espaços constituíam a principal forma de absorção dos egressos das RMPS no mundo do trabalho quando existia a carreira de médico sanitarista, extinta no final da década de 1980.

A partir desse período, a principal política de Estado para a saúde, defendida principalmente pelo Ministério da Saúde, prioriza a formação profissional para atender à estratégia Saúde da Família. Tal política coincide com as diretrizes apresentadas pelo Banco Mundial para investimento público na área da saúde em países em desenvolvimento ${ }^{14}$.

Nesse ponto, não se faz crítica ao PSF ou a sua estratégia de formação, mas ao fato de essa ser a principal, senão a única, política pública substancial na saúde para formação profissional. Todo o resto ficou para o mercado e sociedades de especialidade médicas, cujo papel de regulação da formação especializada é governado pela lógica liberal médico-hegemônica, que valoriza o interesse econômico ou corporativo em detrimento do interesse público ${ }^{15}$.

Destaca-se que a RMPS, além de não ser contemplada pela política pública a partir dos anos 1990, também não encontra sustentação naquela que deveria ser a sua sociedade de "especialidade", a Abrasco, pois ela passa a focar a formação na pós-graduação stricto sensu em detrimento da graduação e das especializações lato sensu, como a residência médica.

Com a política de maior incentivo à pesquisa, a Saúde Coletiva cresceu, afirmou-se e foi reconhecida pela Coordenação de Aperfeiçoamento de Pessoal de Nível Superior (Capes) enquanto campo especializado do conhecimento. Por outro lado, os departamentos de Saúde Coletiva (Medicina Preventiva, Medicina Social, Saúde Comunitária, entre outros) desvalorizaram a formação em Saúde Coletiva nas graduações e na especialização.

A definição do que deve ser ensinado de Saúde Coletiva nos cursos de graduação da área da saúde e a proposta de criação de uma graduação em Saúde Coletiva ${ }^{16}$ são questões em aberto e ainda distantes da construção de um consenso. Enquanto isso, o 
dado de realidade é que o ensino de Saúde Coletiva na graduação, principalmente na Medicina, em geral, é de baixa qualidade, provocando muito mais aversão a esse campo do que propriamente estímulo ao desejo de aproximação. E enquanto esse fator não for modificado, pode-se inferir que esse é um dos elementos que contribui para a redução da procura por essa formação, principalmente por médicos.

No que diz respeito especificamente à RMPS, a não prioridade dos departamentos de Saúde Coletiva em relação a essa modalidade de formação e a despreocupação da Abrasco com a trajetória tomada pela residência produziram o quadro de um acentuado anacronismo entre as os requisitos definidos pela CNRM para os programas de RMPS. Assim, passados 25 anos, de 1981 a 2006, em que ocorreram importantes mudanças na estrutura e no funcionamento do sistema de saúde brasileiro, bem como na conformação do campo da Saúde Coletiva, não houve absolutamente nenhuma mudança na proposta de formação na RMPS.

Por outro lado, ao se analisar a organização dos programas de RMPS remanescentes, verificou-se que cada um, em sua medida, vai incorporando o impacto do crescimento do campo da Saúde Coletiva e das mudanças nas políticas de saúde a partir de cada realidade local, seja de produção acadêmica do departamento, seja da realidade do sistema de saúde em que se desenvolve o programa. Essas diferenças, entretanto, podem também ser a expressão de um distanciamento das demandas dos SUS, enquanto sistema nacional, na medida em que cada programa forma um tipo diferente de profissional, de acordo com a linha vigente na instituição e com o conjunto de saberes e práticas que compõem o campo da Saúde Coletiva.

Após analisar os 11 programas de RMPS remanescentes, foi possível construir uma tipologia que caracteriza o conjunto dos programas, como apresentado no Quadro 7. A maioria dos programas (8) oferece uma formação que pode ser classificada genericamente como de Saúde Coletiva lato sensu, enquanto outros dois programas focam suas atividades em Epidemiologia e Saúde Comunitária. Um único programa não pôde ser classificado, por falta de informações.

Dos oito programas classificados como de Saúde Coletiva, observa-se, entretanto, uma subdivisão de acordo com a ênfase desenvolvida a partir da abordagem realizada no segundo ano, de forma que: três programas oferecem ao residente a opção de aprofundar seu trabalho na linha de pesquisa de um docente do departamento em que desenvolve a residência; três outros têm orientação geral da área de política e gestão em saúde, mas dão ênfase na etapa final da residência a áreas como: Vigilância em
Saúde, Educação em Saúde e Análise Institucional; e um faz formação especializada em Saúde Ocupacional. Um único programa aborda temas gerais de Saúde Coletiva no segundo ano da residência.

\section{Quadro 7}

Tipologia dos programas de residência de Medicina Preventiva e Social

\begin{tabular}{|l|l|l|}
\hline Instituições & Abordagem & \multicolumn{1}{|c|}{ Enfase } \\
\hline FM-ABC & Saúde Coletiva & $\begin{array}{l}\text { Escolha do } \\
\text { residente por linha } \\
\text { de pesquisa do } \\
\text { docente }\end{array}$ \\
\hline USP & Saúde Coletiva & $\begin{array}{l}\text { Escolha do } \\
\text { residente por linha } \\
\text { desquisa do }\end{array}$ \\
\hline docente
\end{tabular}

No que diz respeito aos cenários de práticas, verifica-se a predominância de atividades em cenários técnico-assistenciais e técnico-administrativos. Estes, por sua vez, concentram-se no 
nível municipal, seguindo de maneira lógica a forma de implantação do SUS, que teve a descentralização para os municípios como um dos seus eixos estruturantes ${ }^{17}$. Entretanto, a não vivência de experiências em outros níveis de gestão, como o estadual e o federal, assim como em cenários de caráter político-institucional, pode constituir uma lacuna para a compreensão dos papéis e da dinâmica existente entre os diferentes elementos constitutivos do SUS.

Em outras palavras, a perspectiva paroquial com que a maior parte dos programas tem operado dificulta a compreensão sobre o sistema que articula diferentes práticas em saúde, individuais e coletivas, e que exige que se reflita sobre, por exemplo, o modelo de Estado necessário ao SUS e a inteligência burocrática estatal necessária para operar esse sistema, entre outros temas.

Constata-se, dessa maneira, que a estrutura dos programas de RMPS, em geral, está obsoleta diante das necessidades apresentadas pelo sistema de saúde e da conformação da Saúde Coletiva como campo de saberes e práticas dinâmico e em permanente conformação, embora existam iniciativas e experiências inovadoras que mereçam ser destacadas.

\section{CONSIDERAÇÕES FINAIS}

A motivação para desenvolver a investigação sobre a estrutura dos programas de RMPS partiu do interesse dos pesquisadores em compreender o processo de extinção pelo qual passam esses programas. E também em identificar elementos explicativos do pequeno interesse de acadêmicos e gestores ligados à Saúde Coletiva em intervir sobre o presente processo e formular políticas específicas para este tipo de formação.

Pelas dessemelhanças observadas entre os programas de RMPS, evidenciou-se marcada ausência de diretrizes nacionais que qualifiquem esta residência, incorporando os avanços construídos no campo da Saúde Coletiva, e desenvolvam mecanismos de fortalecimento das interfaces com as necessidades apresentadas pelo SUS.

A partir da análise realizada, inferimos que se trata de um paradoxo resultante de um conjunto de fatores, sobretudo da ausência de uma política de Estado que busque formar e incorporar quadros técnicos com formação específica nas diferentes esferas administrativas e mesmo nos serviços de saúde.

Por outro lado, é importante ressaltar o pequeno interesse e isenção do comprometimento de sujeitos coletivos - como Departamentos de Saúde Coletiva, Abrasco, Comissão Nacional de Residência Médica e gestores públicos na área da saúde - com a formação lato sensu em Saúde Coletiva. A continuidade desse não comprometimento certamente aponta para o processo de extinção das RMPS, ainda que existam elementos para se apostar na residência em Saúde Coletiva, como a necessidade de quadros técnicos de diferentes áreas profissionais, inclusive médicos, com formação lato sensu em Saúde Coletiva para atuação em diferentes cenários do SUS; a necessidade de uma etapa intermediária de formação no campo da Saúde Coletiva entre a graduação e a pós-graduação stricto sensu; e a potência pedagógica do modelo de residência médica ${ }^{18}$.

Endossamos, conclusivamente, o pedido da maior parte dos coordenadores dos programas de RMPS aos formadores e formuladores de políticas públicas de que assumam o compromisso ético-político com a construção do Sistema Único de Saúde brasileiro, colocando em suas agendas a conformação de diretrizes para a formação em diferentes níveis da Saúde Coletiva. Sobretudo da residência médica, que, além de ser um momento de formação, também é um dispositivo que atua na construção dos sistemas de saúde, devido à grande potência com que institui práticas de cuidado e cura.

\section{REFERÊNCIAS}

1. Barros NF, Massuda A, Morita M, Esteves RJF, Guimarães VL. Relatório de pesquisa: Caracterização dos Programas de Residências de Medicina Preventiva e Social no Brasil RMPS, 2007. Observatório de Recursos Humanos em São Paulo. http:/ / www.observarhsp.org.br/projetos/formacao.html.

2. Campos FE, Girardi SN. Caracterização dos Programas de Residência de Medicina Preventiva e Social no Brasil. In: Associação Brasileira de Pós-graduação em Saúde Coletiva. Ensino da Saúde Pública, Medicina Preventiva e Social no Brasil. Rio de Janeiro; 1984.v.3

3. Elias PE, Marsiglia R, Gonçalves RB. Contribuições para o debate sobre Residência em Medicina Preventiva e Social no Brasil. In: Associação Brasileira de Pós-graduação em Saúde Coletiva. Rio de Janeiro; 1988. (Estudos de Saúde Coletiva, v. 5).

4. Escorel S. Reviravolta na Saúde: origem e articulação do movimento sanitário. Rio de Janeiro: Fiocruz; 998.

5. Elias PM. Residência médica no Brasil: a institucionalização da ambivalência. São Paulo; 1987. Mestrado [Dissertação] - Universidade de São Paulo.

6. Arouca ASS. O dilema preventivista: contribuição para a compreensão e crítica da medicina preventiva. São Paulo: Unesp; Rio de Janeiro: Fiocruz; 2003.

7. Nunes ED. A Medicina Social no Brasil, um estudo de sua trajetória. In: Associação Brasileira de Pós-graduação em 
Saúde Coletiva. Estudos de Saúde Coletiva. Rio de Janeiro; 1988. v. 5.

8. Paim J. Desenvolvimento teórico-conceitual do ensino da Saúde Coletiva. In: Associação Brasileira de Pós-graduação em Saúde Coletiva. Ensino da Saúde Pública, Medicina Preventiva e Social no Brasil. Rio de Janeiro; 1982.

9. Buss PM. A experiência do programa de apoio às residências em Medicina Social, Medicina Preventiva e Saúde Pública (PAR MS/MP/SP). In: Associação Brasileira de Pós-graduação em Saúde Coletiva. Ensino da Saúde Pública, Medicina Preventiva e Social no Brasil. Rio de Janeiro; 1982. v.1

10. Campos FE; Belisário AS. O Programa de Saúde da Família e os desafios para a formação profissional e a educação continuada. Interface Comun Saúde Educ. 2001; 133-142.

11. Comissão Nacional de Residência Médica. Relatório Final: A Especialidade Médica no Campo da Saúde Coletiva; 1999. Disponível em:http://www.datasus.gov.br/cns/documentos/relatorio.pdf.

12. Donnangelo MC. A pesquisa na área da saúde coletiva no Brasil: a década de 70. In: Associação Brasileira de Pós-graduação em Saúde Coletiva. Ensino da Saúde Pública, Medicina Preventiva e Social no Brasil. Rio de Janeiro; 1983. v. 2.

13. Elias PEM. Estado e Saúde: Os desafios do Brasil contemporâneo. São Paulo Perspec. 2004; 18(3): 41-46.

14. Rizzotto MLF. As propostas do Banco Mundial para as reformas do setor de Saúde no Brasil nos anos 1990. Saúde Debate. 2005;29(70):140- 7.

15. Campos GWS. Análise crítica sobre especialidades médicas e estratégias para integrá-las ao SUS. Cad Saúde Pública. 1997;13(1):141-4.
16. Teixeira CF. Graduação em Saúde Coletiva: antecipando a formação do sanitarista. I Interface Comun Saúde Educ. 2003;7(13):163-6.

17. Campos GWS. O SUS entre a tradição dos sistemas nacionais e o modo liberal-privado para organizar o cuidado à saúde. Ciênc Saúde Colet. 2007;12(Supl.):1865-74.

18. Feuerwerker LCM. Mudanças na educação médica e residência médica no Brasil. Interface Comun Saúde Educ. 1998;2(3).

19. Campos GWS. Saúde pública e saúde coletiva: campo e núcleo de saberes e práticas. Ciênc Saúde Colet. 2000;5(2):219-30.

20. Paim JS, Almeida Filho N. Saúde coletiva: uma "nova saúde pública" ou campo aberto a novos paradigmas? Rev Saúde Pública. 1998;32(4):299-316.

\section{CONTRIBUIÇÃO DOS AUTORES}

Todos os autores participaram igualmente da revisão bibliográfica, pesquisa e redação. A redação final ficou a cargo de Adriano Massuda e Nelson Felice de Barros

\section{CONFLITO DE INTERESSES}

Declarou não haver

\section{ENDEREÇO PARA CORRESPONDÊNCIA}

Unicamp - Faculdade de Ciência Médicas

Departamento de Medicina Preventiva e Social

Rua Tessália de Camargo, 126 - Cidade Universitária Zeferino Vaz

13083-970 - Campinas - SP

Cx P 16111 